\title{
The future is not always open
}

\author{
James D. E. Grant ${ }^{1} \cdot$ Michael Kunzinger $^{2}$ (1) $\cdot$ Clemens Sämann $^{2}$. \\ Roland Steinbauer ${ }^{2}$
}

Received: 24 January 2019 / Revised: 4 July 2019 / Accepted: 4 September 2019 /

Published online: 12 September 2019

(c) The Author(s) 2019

\begin{abstract}
We demonstrate the breakdown of several fundamentals of Lorentzian causality theory in low regularity. Most notably, chronological futures (defined naturally using locally Lipschitz curves) may be non-open and may differ from the corresponding sets defined via piecewise $C^{1}$-curves. By refining the notion of a causal bubble from Chruściel and Grant (Class Quantum Gravity 29(14):145001, 2012), we characterize spacetimes for which such phenomena can occur, and also relate these to the possibility of deforming causal curves of positive length into timelike curves ( $p u s h$ - up). The phenomena described here are, in particular, relevant for recent synthetic approaches to low-regularity Lorentzian geometry where, in the absence of a differentiable structure, causality has to be based on locally Lipschitz curves.
\end{abstract}

Keywords Causality theory $\cdot$ Low regularity $\cdot$ Chronological future $\cdot$ Causal bubbles

Mathematics Subject Classification 53C50 - 83C75

This work was supported by research grant P28770 of the Austrian Science Fund FWF, and WTZ Project No. CZ 12/2018 of OeAD.

$凶 \quad$ Michael Kunzinger

michael.kunzinger@univie.ac.at

James D. E. Grant

j.grant@surrey.ac.uk

Clemens Sämann

clemens.saemann@univie.ac.at

Roland Steinbauer

roland.steinbauer@univie.ac.at

1 Department of Mathematics, University of Surrey, Guildford, UK

2 Faculty of Mathematics, University of Vienna, Wien, Austria 


\section{Introduction}

Until a decade ago, Lorentzian causality theory had mostly been studied under the assumption of a smooth spacetime metric. However, with the analytic viewpoint on general relativity becoming more and more prevalent, issues of regularity have increasingly come to the fore. In particular, while the geometric core of the theory is traditionally formulated in the smooth category, the analysis of the initial value problem, for example, requires one to consider metrics of low regularity.

Beginning in 2011 with the independent works [8,10], a systematic study of Lorentzian causality theory under low-regularity assumptions began and has brought to light several, sometimes surprising, facts. In a nutshell, while some features of causality theory are rather robust and topological in nature, other results are usually proved by local arguments involving geodesically convex neighbourhoods, which do exist for $\mathcal{C}^{1,1}$-metrics [22,28]. Consequently for this regularity class, the bulk of causality theory $[8,23,28]$ including the singularity theorems $[14,24,25]$ remains valid. Moreover, arguments from causality theory which neither explicitly involve the exponential map nor geodesics have been found to extend to locally Lipschitz metrics (see [8, Thm. 1.25], [29, Rem. 2.5]). However, below $\mathcal{C}^{1,1}$, explicit counterexamples show that convex neighbourhoods may no longer exist for metrics of Hölder regularity $\mathcal{C}^{1, \alpha}$ for any $\alpha<1[19,37]$.

Moreover, Lorentzian causality theory has been generalized to a theory of cone structures, i.e. set-valued maps that assign a cone in the tangent space to each point on a manifold. This setting allows one to develop those aspects of the theory that merely depend on topological arguments under weak regularity assumptions. At the same time, this has led to the introduction of new methods to the field: Using weak KAM theory, existence results for smooth time functions have been derived for $\mathcal{C}^{0}$-cone structures in $[9,10]$, while in [5] dynamical systems theory has been employed to derive similar results for merely upper semi-continuous cone structures. In a landmark paper [29], Minguzzi has extended these studies using methods from set-valued analysis, convexity and order topology to develop large parts of causality theory for locally Lipschitz cone structures.

Returning to the metric spacetime setting, some results, such as the Avez-Seifert theorem, have been extended to $\mathcal{C}^{0}$-Lorentzian metrics [34]. Moreover, a number of fundamental works on the $\mathcal{C}^{0}$-extendibility of spacetimes [12,35,36] have appeared recently. However, already in [8] it was observed that two key features of causality may fail for metrics of regularity below Lipschitz:

(1) The push-up principle, i.e. $\left(I^{+} \circ J^{+}\right) \cup\left(J^{+} \circ I^{+}\right) \subseteq I^{+}$, may cease to hold ${ }^{1}$;

(2) Light cones may cease to be hypersurfaces as they "bubble up" to have a nonempty interior.

Moreover, some fundamental questions raised in [8] remained open, especially the openness of the chronological future $I^{+}(p)$ of a point $p$. In this paper, we answer this question (in the negative) and clarify its relation to points (1) and (2) above. This leads to a number of natural questions concerning the relation between causal and

\footnotetext{
${ }_{1}$ Here $I^{+}$and $J^{+}$denote the chronological and the causal relation, respectively, and e.g. $I^{+} \circ J^{+}=$ $\left\{(p, r) \in M \times M: \exists q \in M\right.$ with $(p, q) \in J^{+}$and $\left.(q, r) \in I^{+}\right\}$.
} 
timelike futures and pasts when defined using various classes of curves (like Lipschitz or piecewise $\mathcal{C}^{1}$ ). To answer these, we construct several counterexamples that exhibit a further breakdown of standard causality properties beyond those already investigated in [8].

Classical accounts on causality theory mainly use piecewise $\mathcal{C}^{1}$-curves. It has to be noted, however, that limit curve theorems, which form a fundamental tool in causality theory, fail to respect this regularity class. This has led Chruściel in [7] to base smooth causality theory entirely on the class of locally Lipschitz curves, an approach that has been adopted in most of the subsequent studies in low regularity. In fact, Chruściel put it as follows in [7, p. 14]:

"In previous treatments of causality theory $[4,13,18,32,33,38]$ one defines future-directed timelike paths as those paths $\gamma$ which are piecewise differentiable, with $\dot{\gamma}$ timelike and future directed wherever defined; at break points one further assumes that both the left-sided and right-sided derivatives are timelike. This definition turns out to be quite inconvenient for several purposes. For instance, when studying the global causal structure of space-times one needs to take limits of timelike curves, obtaining thus - by definition - causal futuredirected paths. Such limits will not be piecewise differentiable most of the time, which leads one to the necessity of considering paths with poorer differentiability properties. One then faces the unhandy situation in which timelike and causal paths have completely different properties. In several theorems, separate proofs have then to be given. The approach we present avoids this, leading we believe - to a considerable simplification of the conceptual structure of the theory."

Besides the above, a strong reason for basing causality theory entirely on locally Lipschitz curves comes from the desire to develop synthetic methods in Lorentzian geometry. In fact, following the pioneering works $[1,17]$, recently methods from synthetic geometry and, in particular, length spaces have been implemented in the Lorentzian setting and in causality theory $[16,21]$. In this framework, which generalizes low-regularity Lorentzian geometry to the level of metric spaces, $\mathcal{C}^{1}$-curves are not available. Thus, one needs a broad enough framework within which to address the problems highlighted by the examples given in Sect. 3 of the present paper.

This article is structured as follows. In Sect. 2, we define the basic notions of causality theory in several variants and establish some fundamental relations between them. We then introduce tools tailored to low regularity, which enable us to characterize openness of chronological futures and pasts, and relate non-bubbling with push-up and clarify several aspects of pathological behaviour of continuous spacetimes. In Sect. 3, we provide explicit continuous spacetimes that display various pathologies. In particular, we give for the first time examples where the chronological future is not open and where the chronological future defined via smooth curves and the one defined via Lipschitz curves differ. This answers several open questions raised in [8]. The final section provides an overview of the interdependence of the various causality notions studied in this work. 
To conclude this introduction, we introduce some basic notation. Throughout this paper, $M$ will denote a smooth connected second countable Hausdorff manifold. Unless otherwise stated, $g$ will be a continuous Lorentzian metric on $M$, and we will assume that $(M, g)$ is time-oriented (i.e. there exists a continuous timelike vector field $\xi$, that is, $g(\xi, \xi)<0$ everywhere). We then call $(M, g)$ a continuous spacetime. We shall also fix a smooth complete Riemannian metric $h$ on $M$ and denote the induced (length) metric by $d_{h}$ and the induced norm by $\|\cdot\|_{h}$. Given Lorentzian metrics $g_{1}, g_{2}$, we say that $g_{2}$ has strictly wider light cones than $g_{1}$, denoted by $g_{1} \prec g_{2}$, if for any tangent vector $X \neq 0, g_{1}(X, X) \leqslant 0$ implies that $g_{2}(X, X)<0$ (cf. [30, Sec. 3.8.2], [8, Sec. 1.2]). Thus, any $g_{1}$-causal vector is timelike for $g_{2}$.

\section{Curves, bubbles, and open questions}

For Lorentzian metrics of regularity at least $\mathcal{C}^{2}$, chronological futures and pasts are usually defined via piecewise $\mathcal{C}^{1}$ (or $\mathcal{C}^{\infty}$ )-curves (e.g. [4,18,30,32]). Especially when working in lower regularity, several authors have employed more general classes of curves, the widest one being that of absolutely continuous curves, basically due to the fact that this is the largest class of functions for which the Fundamental Theorem of Calculus holds. This in turn is required to obtain a reasonable notion of length of curves and to control the curves via their causality. To see this, let $c:[0,1] \rightarrow[0,1]$ be the Cantor function (which has bounded variation) and consider the curve $\gamma:[0,1] \ni$ $t \mapsto(c(t), 0)$ in two-dimensional Minkowski spacetime $\mathbb{R}_{1}^{2}$. Then, $\gamma$ parameterizes the timelike curve $[0,1] \ni t \mapsto(t, 0)$, but its tangent is $(0,0)$ almost everywhere, i.e. spacelike.

Furthermore, absolutely continuous causal curves always possess a locally Lipschitz re-parameterization (cf. Lemma 2.8). On the other hand, irrespective of the regularity of the metric, the limit curve theorems ([27]), which are of central importance in causality theory, even when applied to families of smooth causal curves typically yield curves that are merely locally Lipschitz continuous. It is therefore natural to define causal futures and pasts using such curves, and indeed it was demonstrated in $[7,8,23,28]$ for spacetimes, as well as in the significantly more general setting of cone structures in $[5,10,29]$ that a fully satisfactory causality theory can be based on locally Lipschitz causal curves. As we shall see below, the situation is more involved in the case of timelike curves.

To discuss the dependence of causality theory on the underlying notions of timelike, respectively, causal curves, we introduce the following notations:

\section{Definition 2.1 We denote by}

(1) $\mathcal{A C}$ the set of all absolutely continuous curves from an interval into $M$.

(2) $\mathcal{L}$ the set of all locally Lipschitz curves from an interval into $M$.

(3) $\mathcal{C}_{\mathrm{pw}}^{1}$ the set of all piecewise continuously differentiable curves from an interval into $M$.

(4) $\mathcal{C}^{\infty}$ the set of all smooth curves from an interval into $M$.

Here, a curve is called absolutely continuous if its components in any chart are absolutely continuous, or, equivalently, if it is absolutely continuous as a map into 
the metric space $\left(M, d_{h}\right)$, where we recall that $d_{h}$ is the metric on $M$ induced by the Riemannian metric $h$ on $M$ (cf. the discussion preceding Thm. 6 in [28]).

Clearly, we have

$$
\mathcal{C}^{\infty} \subsetneq \mathcal{C}_{\mathrm{pw}}^{1} \subsetneq \mathcal{L} \subsetneq \mathcal{A C}
$$

Henceforth, when we say that a curve $\gamma$ in $\mathcal{A C}$ is in fact an element of one of these smaller classes, then we mean that there exists a re-parameterization of $\gamma$ that lies in this class.

The definition typically employed in the smooth case (cf. the above references), but sometimes also used for continuous metrics (e.g. in [35]), is the following.

Definition 2.2 Let $\gamma \in \mathcal{C}_{\mathrm{pw}}^{1}$, then $\gamma$ is called

timelike if $g(\dot{\gamma}, \dot{\gamma})<0$ everywhere.

causal if $g(\dot{\gamma}, \dot{\gamma}) \leq 0$ and $\dot{\gamma} \neq 0$ everywhere.

At breakpoints, the understanding is that the above conditions are satisfied for both the one-sided tangents. A causal curve $\gamma$ is called future (past) directed if $\dot{\gamma}$ belongs to the future (past) light cone everywhere (at the breakpoints, this means that both one-sided tangents belong to the same light cone).

The usual definition of causal curves of regularity below piecewise $\mathcal{C}^{1}$ is as follows (cf. [8, Def. 1.3]).

Definition 2.3 Let $\gamma \in \mathcal{A C}$, then $\gamma$ is called

(1) timelike if $g(\dot{\gamma}, \dot{\gamma})<0$ almost everywhere,

(2) causal if $g(\dot{\gamma}, \dot{\gamma}) \leq 0$ and $\dot{\gamma} \neq 0$ almost everywhere.

A causal curve $\gamma$ is called future (past) directed if $\dot{\gamma}$ belongs to the future (past) light cone almost everywhere.

Remark 2.4 In [5], there is one further notion of timelike curve that we should mention: The authors call a Lipschitz curve timelike if its Clarke differential lies in the open chronological cone in the tangent space at each parameter value. They then show ( [5, Lem. 2.11]) that, using this definition, the chronological futures and pasts of a point $p \in M$ are precisely the sets $I_{\mathcal{C}^{\infty}}^{ \pm}(p)$ (see Definition 2.5). Therefore, there is no need for a separate treatment of this approach.

Henceforth, we will adhere to the following convention: Whenever a curve is in $\mathcal{A C}$, we will use Definition 2.3; however, if a curve is explicitly noted to be in $\mathcal{C}_{\mathrm{pw}}^{1}$, then we use Definition 2.2.

We now can define the chronological and causal future and past of a point depending on the class of curves chosen.

Definition 2.5 Let $\mathcal{A} \in\left\{\mathcal{A C}, \mathcal{L}, \mathcal{C}_{\mathrm{pw}}^{1}, \mathcal{C}^{\infty}\right\}$ and let $p \in M$. The chronological and causal future/past of $p$ with respect to $\mathcal{A}$ is

$I_{\mathcal{A}}^{ \pm}(p):=\{q \in M: \exists$ future/past-directed timelike curve $\gamma \in \mathcal{A}$ from $p$ to $q\}$,

$J_{\mathcal{A}}^{ \pm}(p):=\{q \in M: \exists$ future/past-directed causal curve $\gamma \in \mathcal{A}$ from $p$ to $q\} \cup\{p\}$. 
Moreover, for any subset $A \subseteq M$ we set $I_{\mathcal{A}}^{ \pm}(A):=\bigcup_{p \in A} I_{\mathcal{A}}^{ \pm}(p)$ and $J_{\mathcal{A}}^{ \pm}(A):=$ $\bigcup_{p \in A} J_{\mathcal{A}}^{ \pm}(p)$.

From (2.1), we immediately see that for any $p \in M$ we have

$$
\begin{aligned}
& I_{\mathcal{C}^{\infty}}^{ \pm}(p) \subseteq I_{\mathcal{C}_{\mathrm{pw}}^{1}}^{ \pm}(p) \subseteq I_{\mathcal{L}}^{ \pm}(p) \subseteq I_{\mathcal{A} \mathcal{C}}^{ \pm}(p), \\
& J_{\mathcal{C}^{\infty}}^{ \pm}(p) \subseteq J_{\mathcal{C}_{\mathrm{pw}}^{1}}^{ \pm}(p) \subseteq J_{\mathcal{L}}^{ \pm}(p) \subseteq J_{\mathcal{A C}}^{ \pm}(p)
\end{aligned}
$$

Next we recall some key notions from [8] that will be important for our further considerations. For any $C>0$, let $\eta_{C}$ denote the metric $-C \mathrm{~d} t^{2}+\sum_{i=1}^{n}\left(\mathrm{~d} x^{i}\right)^{2}$ on $\mathbb{R}^{n+1}$ (so $\eta \equiv \eta_{1}$ is the Minkowski metric).

Definition 2.6 A chart $\left(\varphi=\left(t, x^{1} \ldots, x^{n}\right), U\right)$ of $M$ centred at $p \in M$ is called cylindrical for $g$ if $U$ is relatively compact, $\varphi(U)=L \times V$ for some interval $L \ni 0$ and $0 \in V \subseteq \mathbb{R}^{n}$ open, and

(i) $\left(\varphi_{*} g\right)(0)=\eta$, the Minkowski metric.

(ii) There exists some $C>1$ such that $\eta_{C^{-1}} \prec \varphi_{*} g \prec \eta_{C}$ on $L \times V$.

Note that point (ii) above implies that $\frac{\partial}{\partial t}$ is timelike and $\frac{\partial}{\partial x^{i}}(1 \leqslant i \leqslant n)$ is spacelike on $U$. By [8, Prop. 1.10], every point $p$ lies in the domain of a cylindrical chart. Such a domain is called a cylindrical neighbourhood.

Following [8, Def. 1.3], an absolutely continuous curve $\gamma$ is called locally uniformly timelike (l.u.t.) if there exists a smooth Lorentzian metric $\stackrel{g}{\prec} g$ such that $\check{g}(\dot{\gamma}, \dot{\gamma})<0$ almost everywhere. For $U \subseteq M$ open and $p \in U$, by $\breve{I}^{ \pm}(p, U)$, we denote the set of all points that can be reached by a future (respectively, past) directed l.u.t. curve in $U$ emanating from $p$. So

$$
\check{I}^{ \pm}(p, U)=\bigcup\left\{I_{\check{g}}^{ \pm}(p, U): \check{g} \in \mathcal{C}^{\infty}, \stackrel{\check{g}}{\prec} \prec\right\}
$$

(with $I_{\grave{g}}^{ \pm}(p, U)$ the chronological future/past of $p$ in $U$ with respect to the smooth Lorentzian metric $\check{g}$ and using smooth curves). In particular, it follows that $\check{I}^{ \pm}(p, U)$ is open.

It was shown in the proof of [8, Prop. 1.10] that in terms of a cylindrical chart $(\varphi, U)$ (where we usually will suppress $\varphi$ notationally), $\partial J_{\mathcal{L}}^{+}(p, U)$ is given as the graph of a Lipschitz function $f_{-}: V \rightarrow L$, that $I_{\mathcal{L}}^{+}(p, U)$ is contained in the epigraph epi $\left(f_{-}\right):=\left\{(t, x): t \geqslant f_{-}(x)\right\}=J_{\mathcal{L}}^{+}(p, U)$ of $f_{-}$and that the interior of $J_{\mathcal{L}}^{+}(p, U)$, denoted by $J_{\mathcal{L}}^{+}(p, U)^{\circ}$, equals the strict epigraph epi ${ }_{S}\left(f_{-}\right):=\left\{(t, x): t>f_{-}(x)\right\}=$ $J^{+}(p, U)^{\circ}$ of $f_{-}$. Here, $f_{-}$is defined as the pointwise limit of the (Lipschitz) graphing functions of $J_{\hat{g}_{k}}^{+}(p, U)$, where the $\hat{g}_{k} \succ g$ are smooth Lorentzian metrics converging locally uniformly to $g$ as $k \rightarrow \infty$. Moreover, the graphing function of $\partial \check{I}^{+}(p, U)$ is denoted by $f_{+}$. 


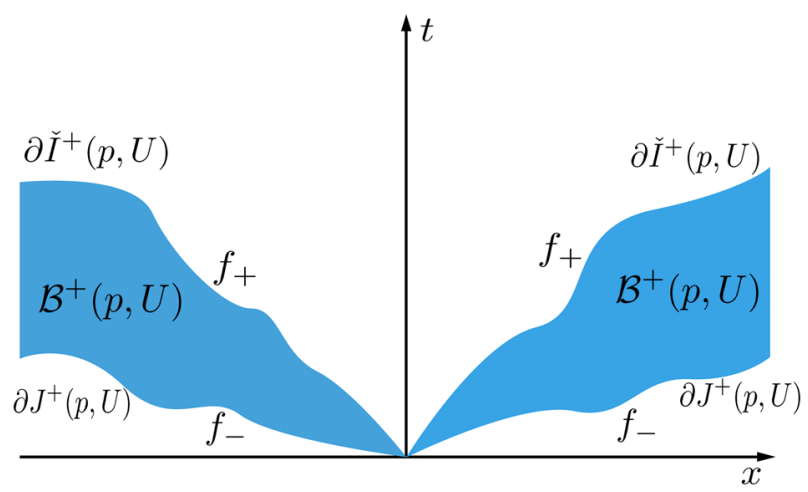

Fig. 1 A cylindrical neighbourhood

Lemma 2.7 For any continuous spacetime $(M, g)$ and any $p \in M$,

$$
\check{I}^{ \pm}(p)=I_{\mathcal{C}^{\infty}}^{ \pm}(p)=I_{\mathcal{C}_{\mathrm{pw}}^{1}}^{ \pm}(p)
$$

In particular, $I_{\mathcal{C}_{\mathrm{pw}}^{1}}^{ \pm}(p)$ is open.

Proof Clearly, $\check{I}^{ \pm}(p) \subseteq I_{\mathcal{C}^{\infty}}^{ \pm}(p) \subseteq I_{\mathcal{C}_{\mathrm{pw}}^{1}}^{ \pm}(p)$. Conversely, let $\gamma:[a, b] \rightarrow M$ be a future-directed timelike piecewise $\mathcal{C}^{1}$ curve from $p$ to some $q \in I_{\mathcal{C}_{\mathrm{pw}}^{1}}^{+}(p)$ and set $c:=\max _{t \in[a, b]} g(\dot{\gamma}(t), \dot{\gamma}(t))<0$ (with the maximum taken over both values at the finitely many breakpoints). Moreover, we have that $\|\dot{\gamma}\|_{h} \leq C^{\prime}$ on $[a, b]$ for some $C^{\prime}>0$. By [8, Prop. 1.2], there exists a smooth Lorentzian metric $\check{g} \prec g$ such that $d_{h}(g, \check{g})<\frac{|c|}{2\left(C^{\prime}\right)^{2}}$, where

$$
d_{h}\left(g_{1}, g_{2}\right):=\sup _{p \in M, 0 \neq X, Y \in T_{p} M} \frac{\left|g_{1}(X, Y)-g_{2}(X, Y)\right|}{\|X\|_{h}\|Y\|_{h}} .
$$

Consequently, $\max _{t \in[a, b]} \breve{g}(\dot{\gamma}(t), \dot{\gamma}(t))<c / 2<0$ for all $t \in[a, b]$, so $q \in I_{\breve{g}}^{+}(p) \subseteq$ $\check{I}^{+}(p)$.

The following Lemma is an analogue of [20, Lem. 3.2.1] (providing a distinguished local parameterization for continuous causal curves in smooth spacetimes) for continuous metrics and cylindrical neighbourhoods:

Lemma 2.8 Let $(M, g)$ be a continuous spacetime, $p \in M$ and let $(\varphi, U)$ be a cylindrical chart around $p$. Let $\gamma:[a, b] \rightarrow U$ be an absolutely continuous futuredirected causal curve. Then there is a re-parameterization $\tilde{\gamma}=\gamma \circ \phi$ of $\gamma$, with $\phi:[c, d] \rightarrow[a, b]$ strictly increasing and absolutely continuous, such that in the chart $\varphi$, for all $t \in[c, d]$ one has

$$
\tilde{\gamma}(t)=(t, \vec{\gamma}(t)) .
$$


Proof Suppressing $\varphi$ notationally, let $C>1$ and $g \prec \eta_{C}$ on $U$. Then $\gamma$ is also future directed with respect to $\eta_{C}$ and hence

$$
0>\eta_{C}\left(\dot{\gamma}, \partial_{t}\right)=-C \dot{\gamma}^{0}
$$

almost everywhere. Thus, $\dot{\gamma}^{0}>0$ almost everywhere, and so $\gamma^{0}$ is strictly monotonically increasing. Setting $\phi:=\left(\gamma^{0}\right)^{-1}:\left[\gamma^{0}(a), \gamma^{0}(b)\right] \rightarrow[a, b]$ and observing that $\dot{\phi}>0$ almost everywhere, we obtain that $\phi$ is absolutely continuous by a result of Zarecki [31, p. 271]. Consequently, $\tilde{\gamma}:=\gamma \circ \phi$ is absolutely continuous by [31, Thm. 3, Ch. IX, $\S 1]$ and so $\tilde{\gamma}$ is a future-directed causal curve with $\tilde{\gamma}(t)=\left(\gamma^{0}\left(\left(\gamma^{0}\right)^{-1}(t)\right), \gamma^{i}(\phi(t))\right)=(t, \vec{\gamma}(t))$ (note that absolutely continuous functions map sets of measure zero to sets of measure zero-Lusin's property, see e.g. [3, Thm. 3.4.3]).

The previous result allows us to conclude that absolutely continuous causal curves always possess a re-parameterization that is Lipschitz continuous. This fact was already noticed in [29, Rem. 2.3]. We include an alternative proof based on cylindrical neighbourhoods for convenience.

Lemma 2.9 Let $(M, g)$ be a continuous spacetime. Then, any causal curve in $\mathcal{A C}$ is locally Lipschitz continuous and hence lies in $\mathcal{L}$. Thus, for any $p \in M, I_{\mathcal{A C}}^{ \pm}(p)=$ $I_{\mathcal{L}}^{ \pm}(p)$ and $J_{\mathcal{A C}}^{ \pm}(p)=J_{\mathcal{L}}^{ \pm}(p)$.

Proof Let $\gamma:[a, b] \rightarrow M$ be an absolutely continuous future-directed causal curve and let $t_{0} \in[a, b]$. Set $p:=\gamma\left(t_{0}\right)$ and let $U$ be a cylindrical neighbourhood of $p$. Moreover, let $\delta>0$ such that $\gamma\left(\left[t_{0}-\delta, t_{0}+\delta\right]\right) \subseteq U$ and let $C>1$ with $g \prec \eta_{C}$ on $U$. Without loss of generality, we can assume that $\gamma(t)=(t, \vec{\gamma}(t))$ on $U$ by Lemma 2.8. Then $\gamma$ is timelike for $\eta_{C}$ and hence

$$
0>\eta_{C}(\dot{\gamma}, \dot{\gamma})=-C+\|\dot{\vec{\gamma}}(t)\|_{e}^{2},
$$

where $\|\cdot\|_{e}$ denotes the Euclidean norm in the chart. This implies that $\|\dot{\gamma}\|_{e} \leq$ $\sqrt{1+C}<\infty$. Consequently, $\dot{\gamma}$ is bounded, and hence, $\gamma$ is Lipschitz continuous.

Based on this result, for $(M, g)$ a continuous spacetime we shall henceforth define the chronological, respectively, causal future/past of a point by $I^{ \pm}(p):=I_{\mathcal{A C}}^{ \pm}(p)=$ $I_{\mathcal{L}}^{ \pm}(p)$ and $J^{ \pm}(p):=J_{\mathcal{A C}}^{ \pm}(p)=J_{\mathcal{L}}^{ \pm}(p)$. This is in accordance with the conventions used in e.g. $[7,8,10,12,14,15,23,34]$, and, for the case of $J^{ \pm}(p)$ also with [5,28,29].

For chronological (i.e. possessing no closed timelike curves) spacetimes, we have the following characterization of openness of chronological futures and pasts:

Theorem 2.10 Let $(M, g)$ be a continuous and chronological spacetime. Then the following are equivalent:

(i) For all $p \in M, I^{ \pm}(p)$ is open.

(ii) For all $p \in M, \partial I^{ \pm}(p)$ is achronal.

(iii) For all $p \in M, \partial I^{ \pm}(p)$ is an achronal Lipschitz-hypersurface. 
(iv) If $F$ is a future/past set with $\partial F \neq \varnothing$, then $\partial F$ is an achronal Lipschitzhypersurface.

\section{Proof $\quad$ (iii) $\Rightarrow$ (ii) is clear.}

(ii) $\Rightarrow$ (i): Suppose that $I^{+}(p)$ were not open for some $p \in M$. Then there exists some $q \in I^{+}(p) \cap \partial I^{+}(p)$. As $M$ is chronological, $p \notin I^{+}(p)$. Since clearly $p \in \overline{I^{+}(p)}$, it follows that $p \in \partial I^{+}(p)$. But then the elements $p$ and $q$ of $\partial I^{+}(p)$ can be connected by a timelike curve, contradicting achronality.

(i) $\Rightarrow$ (iv): Using cylindrical neighbourhoods, this follows exactly as in the smooth case (cf., e.g. [32, Cor. 14.27]), cf. also [11, Proof of Thm. 2.6]. For a detailed proof, see [26, Thm. 2.3.5].

(iv) $\Rightarrow$ (iii): One only has to note that each $\partial I^{+}(p)$ is non-empty, which follows from $M$ being chronological, cf. the argument in (ii) $\Rightarrow$ (i) above.

The spacetime $(M, g)$ is called causally plain ([8, Def. 1.16]) if every $p \in M$ possesses a cylindrical neighbourhood $U$ such that $\partial \check{I}^{ \pm}(p, U)=\partial J^{ \pm}(p, U)$. Otherwise it is called bubbling ${ }^{2}$. Note that whenever we work in a cylindrical chart, all topological notions (like closure, boundary, interior) refer to the relative topology in $U$. If $\partial \check{I}^{ \pm}(p, U) \neq \partial J^{ \pm}(p, U)$, then the open and non-empty set ([8, Prop. 1.10 (vi)])

$$
\begin{aligned}
\mathcal{B}^{+}(p, U) & :=\check{I}^{-}\left(\partial \check{I}^{+}(p, U), U\right) \cap \check{I}^{+}\left(\partial J^{+}(p, U), U\right) \\
& =\left\{(t, x) \in U: f_{-}(x)<t<f_{+}(x)\right\}
\end{aligned}
$$

is called the future bubble set of $p$ (and analogously for the past bubble set), cf. Fig. 1. Refining this terminology, we additionally introduce the interior future bubble set

$$
\mathcal{B}_{\text {int }}^{+}(p, U):=I^{+}(p, U) \backslash \check{I}^{+}(p, U),
$$

as well as the exterior future bubble set

$$
\mathcal{B}_{\text {ext }}^{+}(p, U):=J^{+}(p, U) \backslash \overline{I^{+}(p, U)},
$$

and analogously for the past.

Using Lemmas 2.7 and 2.9, it follows that $\mathcal{B}_{\text {int }}^{+}(p, U)$ consists of those points that can be reached from $p$ by a future-directed Lipschitz timelike curve, but not by a future-directed piecewise $\mathcal{C}^{1}$ timelike curve. It remained an open problem in [8] whether spacetimes with non-trivial interior bubble sets actually exist. We will give examples of this phenomenon in the next section.

The exterior bubbling set is closely connected to push-up properties. To relate these to the concepts introduced above, let us first give a formal definition:

Definition 2.11 A continuous spacetime $(M, g)$ is said to possess the push-up property if the following holds: Whenever $\gamma:[a, b] \rightarrow M$ is a (absolutely continuous) future/past-directed causal curve from $p=\gamma(a)$ to $q=\gamma(b)$ and if $\{t \in[a, b]$ :

\footnotetext{
2 We note that the phenomenon of bubbling, albeit not under this name, was first observed in [6].
} 
$\dot{\gamma}(t)$ exists and is future/past directed timelike $\}$ has nonzero Lebesgue measure, then in any neighbourhood of $\gamma([a, b])$ there exists a future/past-directed timelike Lipschitz curve connecting $p$ and $q$. In particular, $q \in I^{+}(p)$, respectively, $q \in I^{-}(p)$.

This property implies all the commonly used versions of push-up results. In particular, for an absolutely continuous causal curve $\gamma:[a, b] \rightarrow M$ the set $\{t \in[a, b]: \dot{\gamma}(t)$ exists and is future/past directed timelike $\}$ has nonzero Lebesgue measure if and only if $\gamma$ has positive length. A spacetime that possesses the push-up property in the sense of Definition 2.11 also satisfies what in [7, Lemma 2.4.14] and [8, Lemma 1.22] are called push-up Lemma I (i.e. $I^{+}\left(J^{+}(\Omega)\right)=I^{+}(\Omega)$ for any $\Omega \subseteq M$ ) and push-up Lemma II, cf. [7, Lemma 2.9.10] and [8, Lemma 1.24]. The following result shows that push-up is in fact equivalent to the non-existence of exterior bubbling:

Theorem 2.12 Let $(M, g)$ be a continuous spacetime. The following are equivalent:

(i) For each $p \in M$ and each cylindrical chart $U$ centred at $p, \mathcal{B}_{\mathrm{ext}}^{ \pm}(p, U)=\varnothing$.

(ii) For each $p \in M$ and each cylindrical chart $U$ centred at $p, \partial I^{ \pm}(p, U)=$ $\partial J^{ \pm}(p, U)$.

(iii) $(M, g)$ possesses the push-up property.

Proof We will use the facts and notations for cylindrical charts introduced prior to Lemma 2.7.

(i) $\Rightarrow$ (iii): By covering $\gamma([a, b])$ with cylindrical charts $U$ contained in the given neighbourhood of $\gamma([a, b])$, it suffices to show push-up for a curve $\gamma:[a, b] \rightarrow$ $U$ emanating from $p$ that is future-directed causal and such that the set $A:=$ $\{t \in[a, b]: \dot{\gamma}(t)$ exists and is timelike $\}$ has positive Lebesgue measure $\lambda(A)$. Moreover, by Lemma 2.9, we only need to work with Lipschitz curves. So we need to establish that $q:=\gamma(b) \in I^{+}(p, U)$. To begin with, suppose that $q \in$ $J^{+}(p, U)^{\circ}=\operatorname{epi}_{S}\left(f_{-}\right)$. Since $I^{+}(p, U)$ is dense in $J^{+}(p, U)$ by assumption and since $\eta_{C^{-1}} \prec g$ on $U$, we can find an element $q^{\prime} \in I^{+}(p, U)$ such that $q$ lies in the $\eta_{C^{-1}}$-future of $q^{\prime}$, and thereby in $I^{+}(p, U)$ itself. Note that this argument in fact shows that $J^{+}(p, U)^{\circ} \subseteq I^{+}(p, U)$. We are therefore left with the case $q \in \partial J^{+}(p, U)$. Suppose, then, that such a $q$ were not contained in $I^{+}(p, U)$.

We claim that there exists a non-trivial interval $[c, d] \subseteq(a, b)$ such that $\gamma([c, d]) \subseteq$ $I^{+}(p, U)$ and that $\bar{s} \in A$ for some $\bar{s} \in(c, d)$. To see this, we adapt an argument from the proof of [8, Prop. 1.21]. As was shown there, if $t \in A$ and $\gamma(t) \in$ $\partial J^{+}(p, U)$ then there exists some $\varepsilon>0$ such that $\gamma((t, t+\varepsilon))$ lies in $J^{+}(p, U)^{\circ}$ and thereby in $I^{+}(p, U)$ by the above. Arguing analogously to the past, it follows that $\varepsilon$ can be chosen so small that also $\gamma((t-\varepsilon, t))$ is disjoint from $\partial J^{+}(p, U)$ (in fact, lies in $\operatorname{hyp}_{S}\left(f_{-}\right)$). To prove our claim, it suffices to show that there exists some $s \in A$ with $\gamma(s) \notin \partial J^{+}(p, U)$ (hence $\left.\gamma(s) \in \operatorname{epi}_{S}\left(f_{-}\right)\right)$, as then also a non-trivial interval around $s$ is mapped by $\gamma$ to epi ${ }_{S}\left(f_{-}\right)$. So it only remains to exclude the possibility that $\gamma(s) \in \partial J^{+}(p, U)$ for each $s \in A$. To do this, we note that since $\lambda(A)>0, A$ must contain accumulation points of $A$ (otherwise $A$ would consist of isolated points and thereby be countable). However, if $s \in A$ is such an accumulation point and $s_{k}$ is a sequence in $A$ converging to $s$, then choosing $\varepsilon$ as above for $s$ we obtain that $s_{k} \in(s-\varepsilon, s+\varepsilon) \backslash\{s\}$ for $k$ large, contradicting the assumption that $\gamma(A) \subseteq \partial J^{+}(p, U)$. 
Now let $f_{q}^{-}$be the graphing function of $\partial J^{-}(q, U) .{ }^{3}$ Then no point $r$ in $\gamma([c, d])$ can lie strictly below the graph of $f_{q}^{-}$since otherwise $r \in I^{-}(q, U) \cap I^{+}(p, U)$, and a fortiori $q \in I^{+}(p, U)$, contrary to our assumption. Hence, $\left.\gamma\right|_{[c, d]}$ must lie on the graph of $f_{q}^{-}$. But then the same argument as above, using $f_{q}^{-}$and working in the past direction leads to a contradiction since at $\gamma(\bar{s})$ the curve must enter $I^{-}(q, U)$. Summing up, we conclude that $q \in I^{+}(p, U)$, as claimed.

(iii) $\Rightarrow$ (ii): Since $\partial_{t}$ is future directed timelike, (iii) implies that $J^{+}(p, U)^{\circ}=$ $\operatorname{epi}_{S}\left(f_{-}\right) \subseteq I^{+}(p, U)$, so $\partial I^{+}(p, U)=\overline{I^{+}(p, U)} \backslash I^{+}(p, U)^{\circ} \subseteq \partial J^{+}(p, U)$. Conversely, considering a vertical line through any point $q$ in $\partial J^{+}(p, U)$ and using push-up again, it follows that in any neighbourhood of $q$ there lie points from $I^{+}(p, U)^{\circ}$ as well as points from the complement of $\overline{I^{+}(p, U)}$, so $q \in \partial I^{+}(p, U)$. (ii) $\Rightarrow(\mathrm{i})$ : Since $J^{+}(p, U)=\operatorname{epi}\left(f_{-}\right)=\overline{\operatorname{epi}_{S}\left(f_{-}\right)}=\overline{J^{+}(p, U)^{\circ}}$, it suffices to show that $J^{+}(p, U)^{\circ} \subseteq I^{+}(p, U)$. Suppose, to the contrary, that there exists some $q \in J^{+}(p, U)^{\circ} \backslash I^{+}(p, U)$. We can then connect $q$ via a continuous path $\alpha$ that runs entirely in $J^{+}(p, U)^{\circ}$ with a point in $I^{+}(p, U)$ (e.g. with any point on the positive $t$-axis). But then $\alpha$ has to intersect $\partial I^{+}(p, U)$, producing a point in $\partial I^{+}(p, U) \backslash \partial J^{+}(p, U)$, a contradiction.

Remark 2.13 We note that the equivalence between the push-up property and the absence of causal bubbles was first observed, even in the more general setting of closed cone structures (with chronological futures/pasts defined via piecewise $\mathcal{C}^{1}$-curves) by E. Minguzzi in [29, Thm. 2.8].

Concerning the relationship between the various bubble sets, we have:

Lemma 2.14 Let $(M, g)$ be a continuous spacetime and let $U$ be a cylindrical neighbourhood of $p \in M$. Then

$$
\begin{aligned}
& \left\{f_{-}(x)<t<f_{+}(x)\right\} \\
& \quad=\mathcal{B}^{+}(p, U) \subseteq \overline{\mathcal{B}_{\text {int }}^{+}(p, U)} \cup \mathcal{B}_{\text {ext }}^{+}(p, U) \subseteq\left\{f_{-}(x) \leqslant t \leqslant f_{+}(x)\right\},
\end{aligned}
$$

and analogously for the past-directed case.

Proof By [8, Prop. 1.10] and [8, Prop. 1.21], the bubble set $\mathcal{B}^{+}(p, U)$ is the intersection of $\operatorname{epi}_{S}\left(f_{-}\right)$and the strict hypograph hyp ${ }_{S}\left(f_{+}\right)$of the graphing function $f_{+}$ of $\partial \check{I}^{+}(p, U)$. From this, the second inclusion follows immediately. To see the first one, note that by the properties of cylindrical neighbourhoods detailed before Lemma 2.7, any $p \in \mathcal{B}^{+}(p, U)$ is contained in $J^{+}(p, U) \backslash \overline{\check{I}^{+}(p, U)}$. Now suppose, in addition, that $p \notin \overline{\mathcal{B}_{\text {int }}^{+}(p, U)} \supseteq \overline{I^{+}(p, U)} \backslash \overline{I^{+}(p, U)}$. Then $p \in J^{+}(p, U) \backslash \overline{I^{+}(p, U)}=$ $\mathcal{B}_{\text {ext }}^{+}(p, U)$.

From Lemma 2.7 and Lemma 2.9, we obtain:

Theorem 2.15 Let $(M, g)$ be a continuous spacetime. The following are equivalent:

\footnotetext{
${ }_{3}$ It follows from the proof of [8, Prop. 1.10] that the cylindrical neighbourhood $U$ of $p$ can be chosen 'locally uniformly' in the sense that each $q \in U$ possesses a cylindrical chart with domain $U$.
} 
(i) For any $p \in M$,

$$
I_{\mathcal{C}^{\infty}}^{ \pm}(p)=I_{\mathcal{C}_{\mathrm{pw}}^{1}}^{ \pm}(p)=I_{\mathcal{L}}^{ \pm}(p)=I_{\mathcal{A C}}^{ \pm}(p)
$$

(ii) There is no internal bubbling, i.e. $\mathcal{B}_{\mathrm{int}}^{ \pm}(p, U)=\varnothing$ for every $p \in M$ and every cylindrical neighbourhood $U$ of $p$.

(iii) $I^{ \pm}(p)=\breve{I}^{ \pm}(p)$ for every $p \in M$.

Corollary 2.16 Let $(M, g)$ be a continuous spacetime. The following are equivalent:

(i) $(M, g)$ is causally plain.

(ii) There is neither internal nor external bubbling, i.e. $\mathcal{B}_{\text {int }}^{ \pm}(p, U)=\mathcal{B}_{\text {ext }}^{ \pm}(p, U)=$ $\varnothing$, for all $p \in M$.

Proof Let $(M, g)$ be causally plain. By [8, Prop. 1.21], we have $\check{I}^{ \pm}(p)=I^{ \pm}(p)$ for all $p \in M$, so there is no interior bubbling. By [8, Lemma 1.22], $(M, g)$ has the push-up property, so, by Theorem 2.12 , we have $\mathcal{B}_{\text {ext }}^{ \pm}(p, U)=\varnothing$.

The other direction follows directly from Lemma 2.14.

Any spacetime with a Lipschitz continuous metric is causally plain by [8, Cor. 1.17], and so, by Corollary 2.16, such a spacetime has neither internal nor external bubbling. Moreover, by Theorem 2.15, for spacetimes without internal bubbling, it does not matter which type of curves is used in the definition of chronological futures and pasts.

After these preparations, we can now formulate the questions that will be addressed in the remainder of this paper. Let $(M, g)$ be a continuous spacetime. The following two questions have already been posed in [8]:

(Q1) Is $I^{ \pm}(p)$ open for each $p$ ?

(Q2) Is $I^{ \pm}(p)=\breve{I}^{ \pm}(p)$ for each $p$ ?

According to Theorem 2.15, (Q2) is equivalent to the question

$\left(\mathrm{Q} 2^{\prime}\right)$ Is it true that, for all $p \in M, I_{\mathcal{C}}^{ \pm}(p)=I_{\mathcal{C}_{\mathrm{pw}}^{1}}^{ \pm}(p)=I_{\mathcal{L}}^{ \pm}(p)=I_{\mathcal{A C}}^{ \pm}(p)$ ?

Obviously, for spacetimes such that (Q2) can be answered affirmatively, the same is true for $(\mathrm{Q} 1)$. It is natural to ask for the converse of this implication:

(Q3) Does an affirmative answer to (Q1) for a given spacetime imply the same for $(\mathrm{Q} 2)$ ?

Heuristically speaking, to call a set a bubble, one would expect it to have a non-empty interior. A natural question that therefore arises is the following.

(Q4) Is $\check{I}^{+}(p)$ dense in $I^{+}(p)$ i.e. is $I^{ \pm}(p) \subseteq \overline{\check{I}^{ \pm}(p)}$, for all $p \in M$ ?

Finally, we address the relation between bubbling and interior bubbling: As noted above, non-bubbling, i.e. causally plain, spacetimes cannot exhibit interior bubbling. On the other hand, in the only currently known examples of bubbling metrics ([8, Ex. 1.11]) there is no interior bubbling, so all bubble sets are exterior (i.e. lie outside of $\left.\overline{I^{+}(p, U)}\right)$. Another natural question is therefore: 
(Q5) Given a non-causally plain spacetime, are all bubble sets of the same type (interior or exterior)?

In the following section, we provide examples that answer all of these questions in the negative.

\section{Counterexamples}

In this section, we present examples of Lorentzian metrics which show that the answers to the questions (Q1)-(Q5) are negative. The metrics are all defined on a two-dimensional domain $\Omega \subseteq \mathbb{R}^{2}$ with coordinates $(t, x)$, and are taken to be of the form

$$
\mathbf{g}:=2\left[-\sin 2 \theta(t, x) \mathrm{d} t^{2}-2 \cos 2 \theta(t, x) \mathrm{d} x \mathrm{~d} t+\sin 2 \theta(t, x) \mathrm{d} x^{2}\right]
$$

for an appropriately chosen function $\theta(t, x)$. Note that this metric has the property that the vectors

$$
v_{1}:=\cos \theta(t, x) \partial_{x}+\sin \theta(t, x) \partial_{t}, \quad v_{2}:=\cos \theta(t, x) \partial_{t}-\sin \theta(t, x) \partial_{x}
$$

are null, and $\mathbf{g}\left(v_{1}, v_{2}\right)=-2$.

Example 3.1 Let $\Omega:=\mathbb{R}^{2}$ and $0<\alpha<1$. We define the function

$$
\theta(t, x) \equiv \theta(x):= \begin{cases}0, & x<-1, \\ \arccos |x|^{\alpha}, & -1 \leqslant x \leqslant 0 \\ \frac{\pi}{2}, & x>0 .\end{cases}
$$

With this function $\theta$, the metric (3.1) is $\alpha$-Hölder continuous, but not Lipschitz. It is smooth away from $x=0$ and $x=-1$. If we wish to confine the non-smooth behaviour of the metric to the $t$-axis alone, we may restrict to the region $x \in(-1, \infty)$. Alternatively, we may simply smooth out the metric near the set $x=-1$.

The light cones of this metric are illustrated in Fig. 2. If we consider a null generator parameterized by its $x$-value leaving a point $p$ with $t(p)=t_{0}, x(p)=-1$ tangent to the $x$-axis, then its tangent vector will be proportional to $v_{1}$ and, as such, $t(x)$ will satisfy the ordinary differential equation 


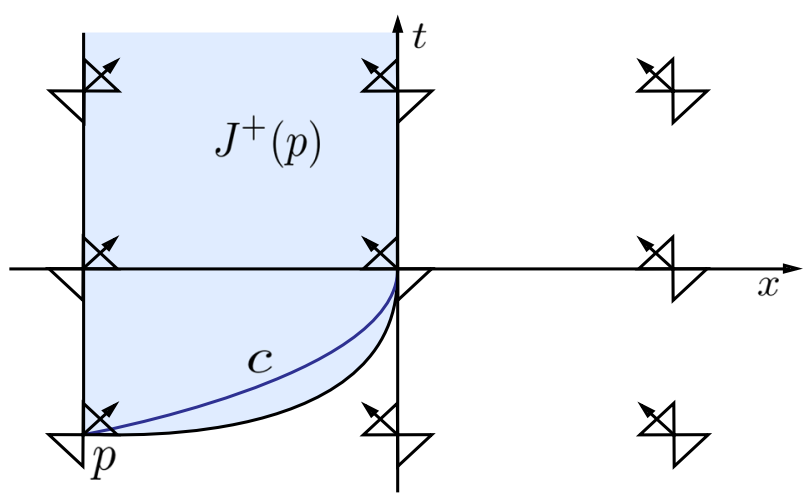

Fig. 2 Timelike curve that reaches $\partial J^{+}(p)$

$$
\frac{\mathrm{d} t}{\mathrm{~d} x}=\tan \theta(t, x)=\frac{\left(1-|x|^{2 \alpha}\right)^{1 / 2}}{|x|^{\alpha}},
$$

for $x \in(-1,0]$ with $t(-1)=t_{0}$. Therefore, the generator will reach the $t$-axis, i.e. $x=0$, at time

$$
t_{1}:=t_{0}+\int_{-1}^{0} \frac{\left(1-|x|^{2 \alpha}\right)^{1 / 2}}{|x|^{\alpha}} \mathrm{d} x<\infty .
$$

Note that this argument crucially relies on the fact that $\alpha<1$. If $g$ were Lipschitz $(\alpha=1)$, for example, the above curve does not reach the $t$-axis in finite time. This is consistent with the fact that Lipschitz metrics are causally plain (cf. Corollary 2.16).

The generator constructed above reaches the $t$-axis at a finite value $t_{1}>t_{0}$ and can then be continued vertically up the $t$-axis as a null generator of $\partial J^{+}(p)$. Indeed, we note that any absolutely continuous, future-directed causal curve from a point on the $t$ axis cannot enter the region $x>0$. In particular, let $\alpha:[0, \infty) \rightarrow \mathbb{R}^{2}$ be an absolutely continuous, future-directed causal curve with $(x \circ \alpha)(0)=0$ and assume that there exists $s_{0}>0$ such that $(x \circ \alpha)\left(s_{0}\right)>0$. Then, there exists a subset $B \subseteq[0, \infty)$ with positive Lebesgue measure such that for all $s \in B, \frac{\mathrm{d}}{\mathrm{d} s}(x \circ \alpha)(s)$ exists and is strictly positive. (If this were not the case, then $(x \circ \alpha)\left(s_{0}\right)=0+\int_{0}^{s_{0}} \frac{\mathrm{d}}{\mathrm{d} s}(x \circ \alpha)(s) \mathrm{d} s \leqslant 0$.) However, any vector of the form $\left(\frac{\mathrm{d} t}{\mathrm{~d} s}, \frac{\mathrm{d} x}{\mathrm{~d} s}\right)$ with $\frac{\mathrm{d} t}{\mathrm{~d} s} \geqslant 0$ and $\frac{\mathrm{d} x}{\mathrm{~d} s}>0$ is not futuredirected causal in the region $x \geqslant 0$. This contradicts the assumption that the curve $\alpha$ is future-directed causal, i.e. that $\dot{\alpha}(s)$ is future-directed causal almost everywhere.

The preceding observation implies that the set $J^{+}(p)$ consists of the light blue region in Fig. 2, along with the vertical null generator from $p$ and the right-moving null generator from $p$. (In particular, the subset of the $t$-axis with $t \geqslant t_{1}$ is part of the boundary of $J^{+}(p)$.)

We now note, however, that any point $(t, 0)$ in the null cone of $p$ with $t>t_{1}$ can also be reached by a curve from $p$ that is $\mathcal{C}^{1}$ and timelike at all points except the intersection of the curve with the $t$-axis. In particular, let $q=(\bar{t}, 0)$ with $\bar{t}>t_{1}$. Then $q \in \partial I^{+}(p)$. Consider the curve $\gamma:(-\varepsilon, 0] \rightarrow M, s \mapsto(t(s), x(s))$ with 


$$
t(s)=\bar{t}+\frac{1}{1-\alpha} A^{1-\alpha} s, \quad x(s)=-A|s|^{\frac{1}{1-\alpha}},
$$

where $A>0$ is constant. Note that $x(s)<0$ for all $s \in(-\varepsilon, 0)$ and $x(0)=0$. Moreover, $\left.\frac{\mathrm{d} t}{\mathrm{~d} s}\right|_{s=0} \neq 0,\left.\frac{\mathrm{d} x}{\mathrm{~d} s}\right|_{s=0}=0$ and, since $\left.\mathbf{g}\right|_{x=0}=4 \mathrm{~d} t \mathrm{~d} x$, it follows that $\frac{d \gamma}{\mathrm{d} s}$ is null at this point. Finally, noting that

$$
\cos \theta(x(s))=|x(s)|^{\alpha}=A^{\alpha}|s|^{\frac{\alpha}{1-\alpha}}, \quad \sin \theta(x(s))=\left(1-A^{2 \alpha}|s|^{\frac{2 \alpha}{1-\alpha}}\right)^{1 / 2}
$$

we now calculate that

$$
\begin{aligned}
\mathbf{g}\left(\frac{\mathrm{d} \gamma}{\mathrm{d} s}, \frac{\mathrm{d} \gamma}{\mathrm{d} s}\right)= & -2\left[2 A^{\alpha}|s|^{\frac{\alpha}{1-\alpha}}\left(1-A^{2 \alpha}|s|^{\frac{2 \alpha}{1-\alpha}}\right)^{1 / 2}\left(-\frac{A^{2}}{(1-\alpha)^{2}}|s|^{\frac{2 \alpha}{1-\alpha}}\right)\right. \\
& \left.+2\left(A^{2 \alpha}|s|^{\frac{2 \alpha}{1-\alpha}}-1\right) \frac{A^{2-\alpha}}{(1-\alpha)^{2}}|s|^{\frac{\alpha}{1-\alpha}}\right] \\
= & -\frac{4}{(1-\alpha)^{2}} A^{2-\alpha}|s|^{\frac{\alpha}{1-\alpha}}\left[2 A^{2 \alpha}|s|^{\frac{2 \alpha}{1-\alpha}}-1+\left(1-A^{2 \alpha}|s|^{\frac{2 \alpha}{1-\alpha}}\right)^{3 / 2}\right] \\
= & -\frac{4}{(1-\alpha)^{2}} A^{2-\alpha}|s|^{\frac{\alpha}{1-\alpha}}\left[\frac{1}{2} A^{2 \alpha}|s|^{\frac{2 \alpha}{1-\alpha}}+O\left(|s|^{\frac{4 \alpha}{1-\alpha}}\right)\right] .
\end{aligned}
$$

As such, the curve $\gamma$ is timelike for small $s<0$ and null only at $s=0$. It follows that, for small $s_{0}<0$, the point $\gamma\left(s_{0}\right)$ lies in $I^{+}(p)$. Since the metric $g$ is smooth for $x<0$, there exists a $\mathcal{C}^{1}$ timelike curve from $p$ to $\gamma\left(s_{0}\right)$. We may assume, without loss of generality, that the tangent vector of this curve coincides with that of the curve $\gamma$ at the point $\gamma\left(s_{0}\right)$. Concatenating this curve with the restriction of $\gamma$ to $\left(s_{0}, 0\right]$ gives a curve $c:[-1,0] \rightarrow \mathbb{R}^{2}$ with $c(-1)=p, c(0)=q \in \partial J^{+}(p)$, that is, $\mathcal{C}^{1}$ with timelike tangent vector on the open interval $(-1,0)$ and whose tangent vector at $q$ is null.

This example has the following properties:

- The chronological future of any point with $x<0$ is not an open subset of $\mathbb{R}^{2}$. In particular, $I^{+}(p)$ consists of the light blue region in Fig. 2, including the subset of the $t$-axis with $t>t_{1}$. This answers (Q1) in the negative.

- $\check{I}^{+}(p)$ consists of the light blue region in Fig. 2, excluding the subset of the $t$-axis with $t>t_{1}$. As such, $\check{I}^{+}(p) \neq I^{+}(p)$. This answers (Q2) in the negative.

- The exceptional curve $c$ constructed in the example may be taken to be smooth, with only one point at which it is null. It may be extended in a timelike fashion to give a curve that is smooth except at one point of non-differentiability, and timelike everywhere where its derivative is defined (at which point, the tangent vector from the past direction is null and the tangent vector to the future is timelike). As such, the curve is not timelike in the sense of Definition 2.2, but it is timelike in the sense of Definition 2.3.

- Since $\breve{I}^{+}(p)$ is not equal to $I^{+}(p)$, it follows from Theorem 2.15 and Corollary 2.16 that the spacetime cannot be causally plain, so there must exist a point $r \in M$ with $\mathcal{B}^{ \pm}(r, U) \neq \varnothing$, for some cylindrical neighbourhood $U$ of $r$. Indeed, while the 


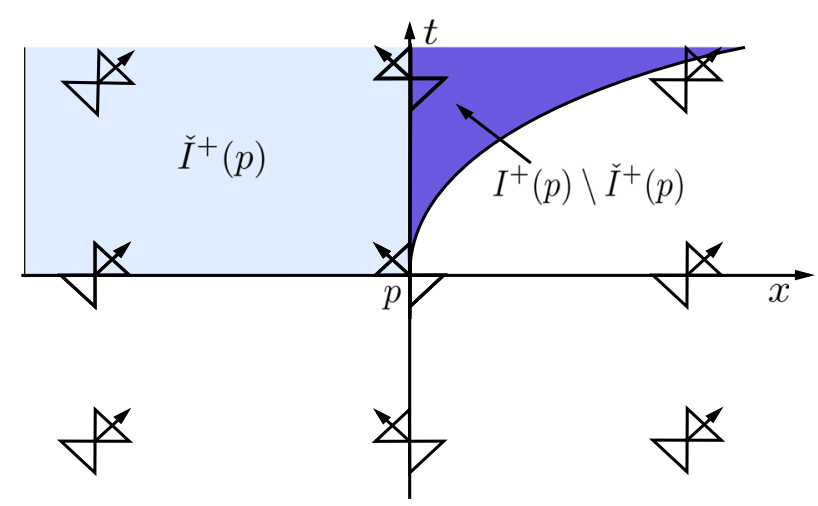

Fig. 3 Interior bubbling

bubble set of $p$ is empty, every point on the $t$-axis has a non-empty past bubble set. This behaviour will be even more pronounced in the following example.

Example 3.2 Let $(t, x) \in \Omega=\mathbb{R}^{2}$, and $0<\alpha<1$. We take the metric to be of the form (3.1), but with

$$
\theta(t, x) \equiv \theta(x):= \begin{cases}0, & x<-1 \\ \arccos |x|^{\alpha}, & -1 \leqslant x \leqslant 1 \\ 0, & x>1\end{cases}
$$

As in Example 3.1, the metric (3.1) is $\alpha$-Hölder but not Lipschitz, and smooth away from $x=0, \pm 1$.

The light cone structure for this metric is illustrated in Fig. 3. For the point $p$ in the figure, the light blue set is the set $\check{I}^{+}(p)$, while the union of the light blue and dark blue sets (including all points on the $t$-axis above the point $p$ ) is the set $I^{+}(p)$. As such, non-trivial internal bubbling occurs for this metric. In particular,

- The set $I^{+}(q)$ is open for all points $q \in \mathbb{R}^{2}$. However, for the particular point $p$ in Fig. 3, we have that $I^{ \pm}(p) \neq \check{I}^{ \pm}(p)$. This shows that the answer to (Q3) is negative.

- For the point $p$ in Fig. 3, the set $\check{I}^{+}(p)$ is contained in the region $x<0$, while $I^{+}(p)$ contains a subset of the region $x>0$ with non-empty interior. It follows that $\check{I}^{+}(p)$ is not a dense subset of $I^{+}(p)$ in this spacetime. Therefore, this example answers (Q4) in the negative.

Example 3.3 In [8], the $\mathcal{C}^{0, \lambda}$ metric

$$
\mathbf{g}=-\left(\mathrm{d} u+\left(1-|u|^{\lambda}\right) \mathrm{d} x\right)^{2}+\mathrm{d} x^{2}
$$

for $(u, x) \in \mathbb{R}^{2}$ with $0<\lambda<1$ was considered, which exhibits external bubbling. In our next example, we define a different metric where the external bubbling effect is localized. We then glue this metric into Example 3.2 above to construct a metric 
where there exists a point $p \in M$ such that $\check{I}^{+}(p) \subsetneq I^{+}(p)$ and $\overline{I^{+}(p)} \subsetneq J^{+}(p)$ at the same time.

Let $x \in \mathbb{R}, t \in \mathbb{R}$, and $0<\alpha, \lambda<1$. Let $\theta_{0}:=\arccos \left(\frac{1}{2}\right)^{\alpha}$, and fix $\rho \in(0,1)$ such that $\arctan \rho^{\lambda}<\theta_{0}$. We define the regions

$$
A:=\left\{(t, x) \in[-\rho, \rho] \times\left[-\frac{5}{6},-\frac{2}{3}\right]\right\}, \quad B:=\left\{(t, x) \in[-1,1] \times\left[-1,-\frac{1}{2}\right]\right\} .
$$

We consider the Lorentzian metric (3.1) where the function $\theta(t, x)$ is defined as follows:

- For $(t, x) \notin B$, we define

$$
\theta(t, x):= \begin{cases}0, & x<-1 \\ \arccos |x|^{\alpha}, & -1 \leqslant x \leqslant 1 \\ 0, & x>1\end{cases}
$$

- For $(t, x) \in A$, let

$$
\theta(t, x):=\arctan |t|^{\lambda}
$$

- We choose the function $\theta$ on the set $B \backslash A$ in such a way that

(i) $\theta$ is continuous on $M \equiv \mathbb{R}^{2}$;

(ii) For each fixed $t \in[-1,1]$, the angle $\theta(t, x)$ is a non-decreasing function of $x \in[-1,0]$, i.e. for each fixed $t \in[-\rho, \rho]$, the function $x \mapsto \theta(t, x)$ is non-decreasing;

(iii) The light cones match up to those on the boundary of the sets $A$ and $B$. In particular, we require

$$
\theta(t,-1)=0, \quad \theta\left(t,-\frac{1}{2}\right)=\arccos \left(\frac{1}{2}\right)^{\alpha}, \quad \forall t \in[-1,1],
$$

and

$$
\theta\left(t,-\frac{5}{6}\right)=\theta\left(t,-\frac{2}{3}\right)=\arctan |t|^{\lambda}, \quad \forall t \in[-\rho, \rho]
$$

Remark 3.4 The function $\theta(t, x)$ may be chosen to be smooth away from the $t$-axis and the points of the set $A$ that lie on the $x$-axis. Globally, we then have that $\theta$ has Hölder regularity $\mathcal{C}^{0, \beta}$, with $\beta:=\min (\alpha, \lambda)$.

The right-moving null generators leaving a point $t=0, x \in\left(-\frac{5}{6},-\frac{2}{3}\right)$ in the region $A$ satisfy

$$
\frac{\mathrm{d} t}{\mathrm{~d} x}=|t|^{\lambda} .
$$




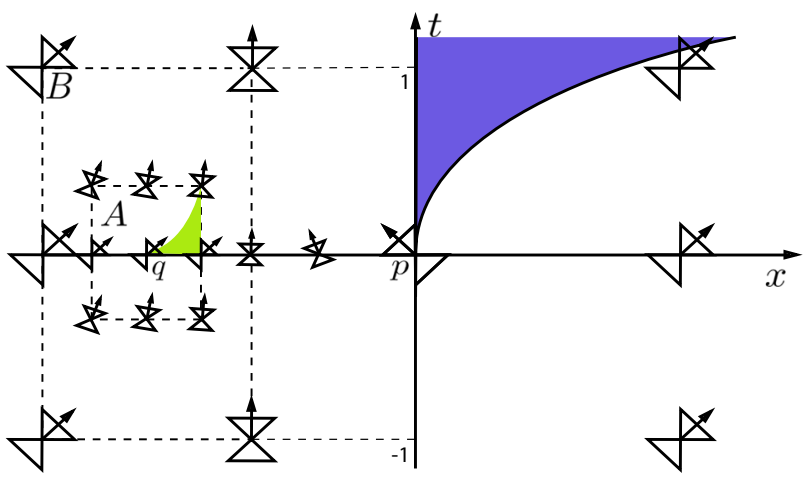

Fig. 4 Parts of the internal and external bubbles of $p$ and $q$, respectively, in Example 3.3

The non-uniqueness of the solutions to this equation leads to the same external bubbling effect demonstrated by metric (3.2) (cf. [8]). In our case, however, this non-uniqueness effect is localized within the region $A$ (Fig. 4).

Let $q=\left(0, x_{0}\right)$ be a point in the interior of the set $A$. We denote by $\gamma_{0}$ the rightmoving null generator from $q$ that immediately leaves the $x$-axis at $q$, i.e. $t\left(\gamma_{0}(s)\right)>0$ for all $s>0$. However, right-moving null generators from $q$ can travel along the $x$ axis for a finite time and then leave the $x$-axis. We denote by $\gamma_{1}$ the right-moving null generator from $q$ that leaves the $x$-axis at the largest value of $x$, i.e. $\gamma_{1}$ leaves the $x$-axis at the point $\left(0, x_{1}\right)$ where $x_{0}<-\frac{2}{3} \leqslant x_{1}<-\frac{1}{2}$. The null generators $\gamma_{0}, \gamma_{1}$ later intersect the $t$-axis at the points $\left(t_{0}, 0\right),\left(t_{1}, 0\right)$, respectively, where $t_{1}<t_{0}$. The set $J^{+}(q) \backslash \overline{I^{+}(q)}$ contains the area between $\gamma_{0}$ and $\gamma_{1}$ up to the $t$-axis and hence has non-empty interior. As in Example 3.2, for any $\bar{t}>t_{0}$, the point $(\bar{t}, 0) \in I^{+}(q)$ and $I^{+}(\bar{t}, 0)$ contains a subset of the set $x>0$ with non-empty interior. Since $\check{I}^{+}(q)$ is contained in the set $x<0$, it follows that $I^{+}(q) \backslash \check{I}^{+}(q)$ has non-empty interior as well.

This example therefore answers (Q5) in the negative and indeed shows that there exist points $q$ in the spacetime such that $\mathcal{B}_{\text {ext }}^{+}(q, U)$ is non-empty for some cylindrical neighbourhood $U$ of $q$, and $\check{I}^{+}(q) \neq I^{+}(q)$.

Remark 3.5 In all of the above examples, the exceptional curves can, in fact, be chosen to be $\mathcal{C}^{1}$ with non-timelike tangent vector at only one point. Heuristically, one might expect that the seemingly innocuous change of allowing $\mathcal{C}^{1}$ curves that have timelike tangent vector except at a finite number of points at which the tangent vector may be null should not affect chronological pasts and futures. However, our examples show that in fact all of the pathologies exhibited above would persist if this definition were adopted.

\section{Conclusions}

The following diagram collects the (non-)implications between the causal regularity properties we have studied in the previous sections. Let $(M, g)$ be a continuous spacetime and recall that $I^{ \pm}(p):=I_{\mathcal{A} \mathcal{C}}^{ \pm}(p)=I_{\mathcal{L}}^{ \pm}(p)$. Then: 


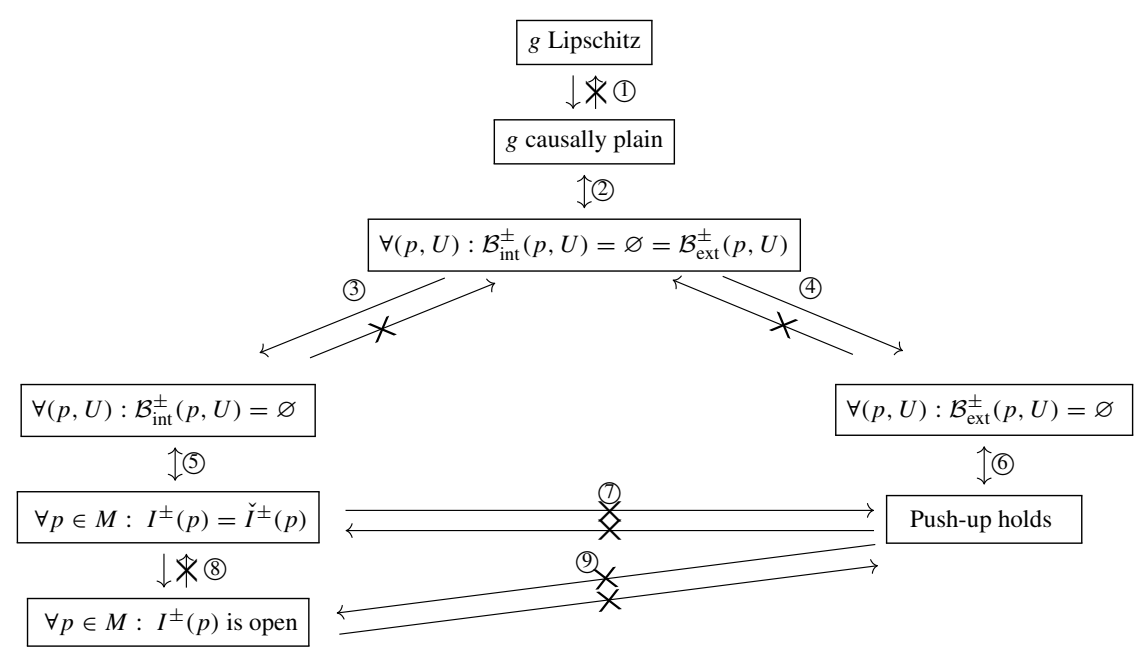

Here, (1) was shown in [8, Cor. 1.17]. Irreversibility of this arrow can be seen by considering a metric that is conformally equivalent to the Minkowski metric via a non-Lipschitz factor. Of more interest is the fact that warped products with onedimensional base (i.e. metrics of the form $-\mathrm{d} t^{2}+f^{2} h$ with $h$ any Riemannian metric and $f:(a, b) \rightarrow(0, \infty)$ continuous) are causally plain for continuous, not necessarily Lipschitz, warping functions $f$, cf. [2]. Equivalence (2) is Corollary 2.16. Irreversibility in (3), (4) and (8), as well as the claims in (9) follow from [8, Ex. 1.12] and Example 3.2, respectively. Equivalence (5) holds by Theorem 2.15, while (6) is a consequence of Theorem 2.12. The claims in (7) follow from [8, Ex. 1.12] and Example 3.1. Finally, Example 3.2 entails irreversibility of (8).

The watershed in this chain of implications is the case of causally plain spacetimes. Indeed, for such metrics all versions of chronological futures and pasts coincide. Moreover, there are no bubble sets, chronological futures and pasts are open and the standard push-up properties hold. Indeed, it was demonstrated in [21, Sec. 5.1] that strongly causal and causally plain spacetimes form strongly localizable Lorentzian length spaces, implying that their causality theory is optimal in the sense of synthetic low-regularity Lorentzian geometry.

On the other hand, the examples in Sect. 3 demonstrate that continuous but nonLipschitz spacetimes display a number of unexpected new causal phenomena that are entirely absent from the causally plain setting. The most drastic of these is the occurrence of non-open chronological futures/pasts. It is no exaggeration to state that openness of $I^{ \pm}(p)$ is ubiquitous throughout standard causality theory. For example, Theorem 2.10 (iv) is a property that features prominently in proofs of the singularity theorems of General Relativity: For the most general version of the causal part of these theorems, we refer to [29, Sec. 2.15] (cf., in particular, the proof of Penrose's singularity theorem [29, Thm. 65] that relies on this property in the form of [29, Thm. 20]). That this condition is (for chronological spacetimes) in fact equivalent to openness of chronological futures/pasts (Theorem 2.10 (i)) is a strong indication 
that it is preferable to adopt a different definition for these sets in regularities below Lipschitz. Indeed, the arguments put forward in this paper suggest that the optimal strategy for obtaining a satisfactory causality theory is the following:

- In order to derive the maximal benefit from limit curve theorems, set $J^{ \pm}(p):=$ $J_{\mathcal{A C}}^{ \pm}(p)=J_{\mathcal{L}}^{ \pm}(p)$ (cf. Lemma 2.9).

- To guarantee openness of chronological futures and pasts and to avoid interior bubbling, set $I^{ \pm}(p):=I_{\mathcal{C}_{\mathrm{pw}}^{1}}^{ \pm}(p)$. Then in fact $I^{ \pm}(p)=\breve{I}^{ \pm}(p)=I_{\mathcal{C}^{\infty}}^{ \pm}(p)$ by Lemma 2.7.

The first point here is valid across all regularities of the metric. For the second one, we have seen in Theorem 2.15 that for spacetimes without internal bubbling (in particular: for Lipschitz spacetimes) the choice of class of curves makes no difference, while the examples in Sect. 3 clearly underline the advantage of adhering to this convention for non-Lipschitz metrics.

While this strategy returns us to the "unhandy situation in which timelike and causal paths have completely different properties" [7, p. 14], it appears to us to be the most fitting approach. Moreover, note that, in fact, several fundamental works on low-regularity Lorentzian geometry have adopted these conventions, in particular $[5,28,29]$ and, in the timelike case, $[11,35]$. On the other hand, in synthetic approaches to low-regularity Lorentzian geometry (such as [16,21]), where this strategy is not an option (due to the absence of a differentiable structure), phenomena such as the ones laid out in Sect. 3 have to be taken into consideration.

Acknowledgements Open access funding provided by Austrian Science Fund (FWF). We are indebted to two anonymous referees for several remarks that have led to considerable improvements of the manuscript.

Open Access This article is distributed under the terms of the Creative Commons Attribution 4.0 International License (http://creativecommons.org/licenses/by/4.0/), which permits unrestricted use, distribution, and reproduction in any medium, provided you give appropriate credit to the original author(s) and the source, provide a link to the Creative Commons license, and indicate if changes were made.

\section{References}

1. Alexander, S.B., Bishop, R.L.: Lorentz and semi-Riemannian spaces with Alexandrov curvature bounds. Commun. Anal. Geom. 16(2), 251-282 (2008)

2. Alexander, S.B., Graf, M., Kunzinger, M., Sämann, C.: Generalized cones as Lorentzian length spaces: Causality, curvature, and singularity theorems (2019) (in preparation)

3. Ambrosio, L., Tilli, P.: Topics on Analysis in Metric Spaces. Oxford Lecture Series in Mathematics and its Applications, vol. 25. Oxford University Press, Oxford (2004)

4. Beem, J.K., Ehrlich, P.E., Easley, K.L.: Global Lorentzian Geometry. Monographs and Textbooks in Pure and Applied Mathematics, vol. 202, 2nd edn. Marcel Dekker Inc., New York (1996)

5. Bernard, P., Suhr, S.: Lyapounov functions of closed cone fields: from conley theory to time functions. Commun. Math. Phys. 359(2), 467-498 (2018)

6. Chruściel, P. T.: On uniqueness in the large of solutions of Einstein's equations ("strong cosmic censorship"). In: Proceedings of the Centre for Mathematics and its Applications, Australian National University 27, Canberra (1991)

7. Chruściel, P. T.: Elements of causality theory (2011). arXiv:1110.6706 [gr-qc]

8. Chruściel, P.T., Grant, J.D.E.: On Lorentzian causality with continuous metrics. Class. Quantum Gravity 29(14), 145001 (2012)

9. Fathi, A.: Time functions revisited. Int. J. Geom. Methods Mod. Phys. 12(8), 1560027 (2015) 
10. Fathi, A., Siconolfi, A.: On smooth time functions. Math. Proc. Camb. Philos. Soc. 152(2), 303-339 (2012)

11. Galloway, G.J., Ling, E.: Some remarks on the $C^{0}$-(in)extendibility of spacetimes. Ann. Henri Poincaré 18(10), 3427-3447 (2017)

12. Galloway, G.J., Ling, E., Sbierski, J.: Timelike completeness as an obstruction to $C^{0}$-extensions. Commun. Math. Phys. 359(3), 937-949 (2018)

13. Geroch, R.: Domain of dependence. J. Math. Phys. 11, 437-449 (1970)

14. Graf, M., Grant, J.D.E., Kunzinger, M., Steinbauer, R.: The Hawking-Penrose singularity theorem for $C^{1,1}$-Lorentzian metrics. Commun. Math. Phys. 360(3), 1009-1042 (2018)

15. Graf, M., Ling, E.: Maximizers in Lipschitz spacetimes are either timelike or null. Class. Quantum Gravity 35(8), 087001 (2018)

16. Grant, J.D.E., Kunzinger, M., Sämann, C.: Inextendibility of spacetimes and Lorentzian length spaces. Ann. Glob. Anal. Geom. 55, 133-147 (2019)

17. Harris, S.G.: A triangle comparison theorem for Lorentz manifolds. Indiana Univ. Math. J. 31(3), 289-308 (1982)

18. Hawking, S.W., Ellis, G.F.R.: The Large Scale Structure of Space-Time. Cambridge Monographs on Mathematical Physics, vol. 1. Cambridge University Press, London (1973)

19. Hartman, P., Wintner, A.: On the problems of geodesics in the small. Am. J. Math. 73, 132-148 (1951)

20. Kriele, M.: Spacetime: Foundations of General Relativity and Differential Geometry. Lecture Notes in Physics. New Series m: Monographs, vol. 59. Springer, Berlin (1999)

21. Kunzinger, M., Sämann, C.: Lorentzian length spaces. Ann. Glob. Anal. Geom. 54(3), 399-447 (2018)

22. Kunzinger, M., Steinbauer, R., Stojković, M.: The exponential map of a $C^{1,1}$-metric. Differ. Geom. Appl. 34, 14-24 (2014)

23. Kunzinger, M., Steinbauer, R., Stojković, M., Vickers, J.A.: A regularisation approach to causality theory for $C^{1,1}$-Lorentzian metrics. Gen. Relativ. Gravity 46(8), 1738 (2014)

24. Kunzinger, M., Steinbauer, R., Stojković, M., Vickers, J.A.: Hawking's singularity theorem for $C^{1,1_{-}}$ metrics. Class. Quantum Gravity 32(7), 075012 (2015)

25. Kunzinger, M., Steinbauer, R., Vickers, J.A.: The Penrose singularity theorem in regularity $C^{1,1}$. Class Quantum Gravity 32(15), 155010 (2015)

26. Lecke, A.: Non-smooth Lorentzian geometry and causality theory. PhD thesis, University of Vienna, Faculty of Mathematics (2016)

27. Minguzzi, E.: Limit curve theorems in Lorentzian geometry. J. Math. Phys. 49(9), 092501 (2008)

28. Minguzzi, E.: Convex neighborhoods for Lipschitz connections and sprays. Monatsh. Math. 177(4), 569-625 (2015)

29. Minguzzi, E.: Causality theory for closed cone structures with applications. Rev. Math. Phys. 31(5), 1930001 (2019)

30. Minguzzi, E., Sánchez, M.: The causal hierarchy of spacetimes. In: Alekseevsky, D.V., Baum, H. (eds.) Recent developments in pseudo-Riemannian geometry, ESI Lectures in Mathematics and Physics, pp. 299-358. European Mathematical Society, Zürich (2008)

31. Natanson, I. P.: Theory of Functions of a Real Variable (Translated by Leo F. Boron with the collaboration of Edwin Hewitt). Frederick Ungar Publishing Co., New York (1955)

32. O’Neill, B.: Semi-Riemannian Geometry with Applications to Relativity. Pure and Applied Mathematics. Academic Press, Inc. [Harcourt Brace Jovanovich, Publishers], New York (1983)

33. Penrose, R.: Techniques of differential topology in relativity. In: Conference Board of the Mathematical Sciences Regional Conference Series in Applied Mathematics, vol. 7, Society for Industrial and Applied Mathematics, Philadelphia (1972)

34. Sämann, C.: Global hyperbolicity for spacetimes with continuous metrics. Ann. Henri Poincaré 17(6), 1429-1455 (2016)

35. Sbierski, J.: The $C^{0}$-inextendibility of the Schwarzschild spacetime and the spacelike diameter in Lorentzian geometry. J. Differ. Geom. 108(2), 319-378 (2018)

36. Sbierski, J.: On the proof of the $C^{0}$-inextendibility of the Schwarzschild spacetime. J. Phys. Conf. Ser. 968(1), 012012 (2018)

37. Sämann, C., Steinbauer, R.: On geodesics in low regularity. J. Phys. Conf. Ser. 968(1), 012010 (2018)

38. Wald, R.M.: General Relativity. University of Chicago Press, Chicago (1984)

Publisher's Note Springer Nature remains neutral with regard to jurisdictional claims in published maps and institutional affiliations. 